\title{
A patient with spotted fever group rickettsiosis mimicking connective tissue disease
}

\author{
R Premaratna1, E W Liyanaarachchi' ${ }^{1}$ R P V J Rajapakse ${ }^{2}$, N K B K R G W Bandara ${ }^{3}$, H J de Silva ${ }^{1}$
}

Ceylon Medical Journal 2012; 57: 127-128

\section{Introduction}

Common clinical features associated with spotted fever group (SFG) rickettsioses include fever, headache, arthralgia, arthritis, gastro-intestinal disturbances, lymphadenopathy, hepato-splenomegaly and a discrete maculo-papular rash [1]. However, diverse clinical manifestations associated with SFG rickettsioses have been reported, some presenting with a continued febrile illness with no definite features suggestive of rickettsioses [2]. We describe a patient with SFG rickettsiosis who had small joint arthropathy, cervical lymphadenopathy, hair loss, oral ulceration, and intermittent high fever over a 4 month period. She was later confirmed of having SFG rickettsioses and all her clinical features resolved with anti-rickettsial antibiotics.

\section{Case report}

A 35-year old married sub-fertile woman presented in April 2009 with high intermittent fever associated with chills and rigors, a dry cough, loss of appetite, lethargy, symmetrical small joint arthropathy, (which had appeared over about two weeks of initial febrile illness and persisted with varying degrees of severity) and painful cervical lymphadenopathy for over 4 months, with worsening of symptoms over the past 10 days. She had undergone many haematological, histological (lymph node biopsy) and biochemical investigations, but no definitive diagnosis had been made. On examination she looked ill, had oral ulcers, but was not pale or icteric and there was no clinical evidence of a vasculitis. Her hair was sparse and thin but there was no scarring alopecia. She had $1-2 \mathrm{~cm}$, tender, posterior deep cervical lymph nodes on the left side and anterior lower deep cervical nodes on the right. There were inspiratory crackles in both lung fields, but abdominal examination was normal (there was no hepatosplenomegaly). Her clinical presentation was suggestive of a connective tissue disorder. The investigation results were as follows; WBC: $2.9 \times 10^{6} / \mu^{1}, \mathrm{~N}-40 \%, \mathrm{~L}-55 \%, \mathrm{Hb}-12.8$ $\mathrm{g} / \mathrm{dl}$, Platelet count- $223 \times 10^{6} / \mu^{1}$, blood picture - normal red cell morphology, numerous reactive lymphocytes but no abnormal cells. ESR 65 mm 1st hour, CRP2.6 (normal 0- 5mg/l), SGPT $28 \mathrm{iu} / 1$, SGOT 32 iu /1, serum LDH $3236 \mathrm{iu} / 1$, normal chest $\mathrm{X}$ ray, normal ultrasound of the abdomen. ANA, anti-DsDNA, HIV antibodies, EBV antibodies, toxoplasma antibodies, and mantoux test were negative. Two lymph node biopsies which had been done within a space of two months showed reactive changes, and was negative for tuberculosis by PCR. Because she was ill and had high fever she was given intravenous ceftriaxone and levofloxacin empirically, but had no clinical improvement over the next 5 days.

As she was having continued high intermittent fever associated with lymphadenopathy, we subsequently tested her for rickettsioses by IFA test using rickettsial antigens prepared from cell culture grown rickettsiae: Rickettsia conorii (Malish) (RC), Rickettsia typhi (Wilmington), and Orientia tsutsugamushi (Karp) (OT). $\mathrm{IgG}$ antibodies were detected using fluoresceinconjugated goat anti-human $\operatorname{IgG}(\gamma)$ and $\operatorname{IgM}$ (KPL, Inc., Gaithersburg, MD) and the serum was strongly positive for $\mathrm{SFG}$ ricketsioses with a dilution titer of $>1: 8192$. She was started on oral doxycycline, but reacted to the drug after 2 days with epigastric pain and vomiting. She was therefore given oral azithromicin and intravenous chloramphenicol to which she showed a good clinical response over three days. When reviewed 6 months and 12 months after leaving hospital she remained asymptomatic with complete regression of lymphadenopathy and oral ulcerations. She had re-grown her hair. Her ANA which was repeated remained negative and the IFA dilution titer using $R$. conorii antigen was 1 : 128 after one year.

\section{Discussion}

This patient with SFG rickettsiosis presented with a clinical picture suggestive of a connective tissue disorder such as systemic lupus erythematosus or a lymphoma, but there was no evidence for these conditions. Infections such as infectious mononucleoses, toxoplasmosis, HIV and tuberculosis were also excluded. She had a good clinical response to a combination of azithromicin and chloramphenicol which have good antirickettsial activity. Medicine, University of Peradeniya, Sri Lanka.

Correspondence: RP, e-mail: <ranjan_premaratna@lycos.com>. Received 18 July 2011 and revised version accepted 29 January 2012. Competing interests: none declared. 
We would like to highlight the importance of being aware of the diverse clinical manifestations of SFG rickettsial infections especially in areas where rickettsial diseases are endemic or re-emerging, in order to prevent morbidity and possible mortality associated with a delay in the diagnosis of these infections [3]. It also helps in reducing direct costs such as cost of extensive investigations and inappropriate treatment and indirect costs due to loss of productive man hours.

\section{References}

1. Parola P, Paddock CD, Raoult D. Tick-borne rickettsioses around the world: emerging diseases challenging old concepts. Clinical Microbioogy Review 2005; 18: 719-56.

2. Premaratna R, Rajapakse RP, Chandrasena TG, et al. Contribution of rickettsioses in Sri Lankan patients with fever who responded to empirical doxycycline treatment. Transaction of Royal Society Tropical Medicine and Hygiene 2010; 104: 368-70.

3. Premaratna R, Loftis AD, Chandrasena TG, Dasch GA, de Silva HJ. Rickettsial infections and their clinical presentations in the Western Province of Sri Lanka: a hospital-based study. International Journal of Infection Diseases 2009; 12: 198-202. 